\title{
SEPARATION PROPERTIES OF THE WALLMAN ORDERED COMPACTIFICATION
}

\author{
D.C. KENT \\ Department of Pure and Applied Mathematics \\ Washington State University \\ Pullman, WA 99164 \\ T.A. RICHMOND \\ Department of Mathematics \\ Western Kentucky University \\ Bowling Green, KY 42101 \\ (Received December 19, 1988 and in revised form February 8, 1990)
}

ABSTRACT. The Wallman ordered compactification $w_{0} X$ of a topological ordered space $X$ is $T_{2}$-ordered (and hence equivalent to the Stone-Cech ordered compactification) iff $X$ is a $T_{4}$-ordered c-space. In particular, these two ordered compactifications are equivalent when $X$ is $n$ dimensional Euclidean space iff $n \leq 2$. When

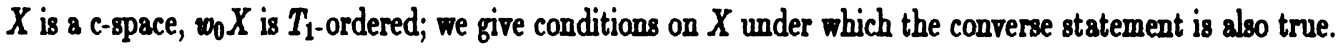
We also find conditions on $X$ which are necessary and sufficient for $w_{0} X$ to be $T_{2}$. Several examples provide further insight into the separation properties of $w_{0} X$.

KEY WORDS AND PHRASES. c-set, maximal c-filter, $T_{1}$-ordered space, $T_{2}$-ordered space, ordered compactification.

1980 AMS SUBJECT CLASSIFICATION CODES. 54F05, 54D35, 54D10

\section{Introduction.}

The Wallman ordered compactification $w_{0} X$ of a $T_{1}$-ordered space $X$ was introduced in 1979 by Choe and Park [1]. In [3] one of the authors showed (in the terminology of this paper) that $w_{0} X$ is $T_{2}$-ordered iff $X$ is a $T_{4}$-ordered c-space, and that for such spaces, $w_{0} X$ is equiralent to the Stone-Cech ordered (or Nachbin) compactification $\beta_{0} X$ of $X$. 
This paper continues the study of the separation properties of $w_{0} X$. If $X$ is a c-space (meaning that the increasing and decreasing hulls of every c-set are closed), then $w_{0} X$ is $T_{1}$-ordered, and under certain further restrictions on $X$ the condition of being a c-space is shown to be necessary in order for $w_{0} X$ to be $T_{1}$-ordered (see Theorems 2.7 and 2.8). Two conditions on $X$ are found which are necessary and sufficient for $w_{0} X$ to be $T_{2}$; one is an ultrafilter condition, while the other is a version of normality for ordered spaces which we call "c-normally ordered." For $T_{1}$-ordered c-spaces, the notions "c-normally ordered" and "normally ordered" (as defined by Nachbin, [5]) are equivalent, but for $T_{1}$-ordered spaces in general it is shown by examples that neither property implies the other.

One motivation for studying the Wallman ordered compactification is that it gives a convenient filter characterization for $\beta_{0} X$ when $X$ is a $T_{4}$-ordered c-space. For Euclidean n-space $R^{n}$, we show that $w_{0} R^{n}$ and $\beta_{0} R^{n}$ are equivalent iff $n \leq 2$, and we then give a description of $\beta_{0} R^{2}$ based on the Wallman characterization of compactification points in $\beta_{0} R^{2}$ as non-convergent maximal c-filters. Other examples are given to show how the separation properties of the Wallman ordered compactification can fail in various ways and combinations.

\section{The Wallman Ordered Compactification.}

If $(X, \leq)$ is a poset and $A$ a non-empty subset of $X$, we define $d(A)=\{y \in X: y \leq x$ for some $x \in A\}$ to be the decreasing hull of $A$; the increasing hull $i(A)$ is defined dually. We shall write $d(x)(i(x))$ in place of $d(\{x\})(i(\{x\}))$. A subset $A$ is increasing (respectively, decreasing) if $A=i(A)$ (respectively, $A=d(A))$. A set which is either increasing or decreasing is said to be monotone. If $A=i(A) \cap d(A)$, then $A$ is called a conver set.

We shall use the term space throughout this paper to mean a triple $(X, \leq, \tau)$, where $(X, \leq)$ is a poset and $r$ a convez toplogy on $X$ (i.e., a topology for which the open monotone sets constitute an open subbase). When there is no danger of confusion, we shall designate the space $(X, \leq, \tau)$ simply by " $X$ ”.

For any space $X$, we shall use the term fundamental open set to mean any set expressible as a finite intersection of finite unions of monotone open sets. The set $U_{X}$ of all fundamental open sets forms an open base for $X$. The complement of a fundamental open set will be called a fundamental closed set.

Let $A$ be a subset of a space $X$, and let $I(A)$ (respectively, $(D(A))$ be the smallest closed and increasing (respectively, closed and decreasing) set containing $A$, and let $A^{\wedge}=I(A) \cap D(A)$. If $A=A^{\wedge}$ then $A$ is called a c-set; let $C_{X}$ denote the collection of all c-sets on a space $X$. One can verify that $C_{X}$ is closed under 
intersections and forms a subbase for the collection of closed sets in a space $X$. The relationship between fundamental open sets and c-sets can be described as follows.

Proposition 1.1 Let $X$ be a space. Then $U \in U_{X}$ iff $X-U$ is a finite union of c-sets.

If $\mathcal{J}$ is a filter on a space $X$, let $I(\mathcal{J})$ be the filter generated by $\{I(F): F \in \mathcal{F}\}$; the filters $D(\mathcal{F}), i(\mathcal{F})$, and $d(\mathcal{F})$ are defined similarly. The fixed ultrafilter generated by an element $x$ in $X$ is denoted by $\dot{x}$. If $\mathcal{I}$ and $\mathcal{G}$ are filters on $X$ which do not contain disjoint sets, let $\xi \vee \mathcal{G}$ designate the filter generated by $\{F \cap G: F \in \mathcal{F}, G \in \mathcal{G}\}$; if $F \cap G=\phi$ for some $F \in \mathcal{F}$ and $G \in \mathcal{G}$, we sas that $₹ \vee G$ "fails to exist" (as a proper filter).

For any filter $\mathcal{F}$, the filter $\xi^{\wedge}=I(\xi) \vee D(\xi)$ exists and is generated by $\left\{F^{\wedge}: \boldsymbol{F} \in \mathcal{J}\right\}$. If $\xi=\xi^{\wedge}$, then $f$ is called a c-filter. It is easy to show (using Zorn's Lemma) that every c-filter is coarser than a maximal c-filter. In our study of the Wallman ordered compactification, which is based on maximal c-filters, the following characterization will be useful.

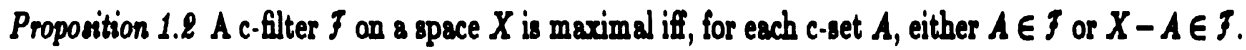

Proof. If $J$ is a maximal $c$-filter and $A \notin J$, then $J$ can have no trace on $A$, for such a trace would be a $c$-filter strictly finer than $A$. Thus $X-A \in \mathcal{J}$. Conversely, if $\mathcal{J}$ is a c- - ilter which is not maximal, and $\mathcal{G}$ is a strictly finer c-filter, then some c-set $G$ in $g$ has the property that neither $G$ nor $X-G$ is in $\mathcal{F}$, contrary to the stated condition. I

A space $X$ is $T_{1}$-ordered $[4]$ if, for each $x \in X, i(x)$ and $d(x)$ are both closed sets. Note that in a $T_{1}$-ordered space, each singleton $\{x\}$ is a c-set. $A$ space with closed order is defined to be $T_{2}$-ordered $[4]$. $A$ space $X$ is normally ordered $[5]$ if, whenever $A$ and $B$ are diajoint closed sets, with $A$ increaing and $B$ decreasing, there are disjoint open sets $U$ and $V$, with $U$ increasing and $V$ decreaing, such that $A \subseteq U$ and $B \subseteq V$. A space which is both normally ordered and $T_{1}$-ordered is said to be $T_{4} \cdot$ ordered $[3]$. Priestly, $[6]$, defined a $C$-space to be one in which $i(A)$ and $d(A)$ are closed whenerer $A$ is closed. We define a cospace to be one in which every c-set $A$ has the property that $i(A)$ and $d(A)$ are closed sets. Obvioualy, every C-space is a c-space; in particular, the compact, $T_{2}$-ordered apaces are c-spaces. An altemate characterisation for c-spaces is given in the next proposition (see [3]).

Proposition 1.9 If $X$ is a c-space and $A, B$ are c-sets, then $I(A) \cap B=\phi$ implies $I(A) \cap D(B)=\phi$, and $D(A) \cap B=\phi$ implies $D(A) \cap I(B)=\phi$. If $X$ is $T_{4}$-ordered and the tro preceding implications bold for arbitrary c-sets $A$ and $B$, then $X$ is a c-space. 
The Wallman ordered compactification can be constructed for any $T_{1}$-ordered space $X$. The original construction by ('hoe and Park [1] was based on "maximal bifilters"; we shall follow the approach of [3] in which maximal c-filters form the underlying set for $w_{0} X$. Given a $T_{1}$-ordered space $X$, let $w_{0} X=\{x: x \in$ $X\} \cup X^{\prime}$, where $X^{\prime}$ is the set of all non-convergent maximal c-filters. A partial order relation is defined for $w_{0} X$ as follows: $\mathcal{F} \mathcal{G}$ iff $I(\mathcal{F}) \subseteq \mathcal{G}$ and $D(\mathcal{G}) \subseteq \mathcal{F}$. The embedding map $\varphi: X \rightarrow w_{0} X$ given by $\varphi(x)=\stackrel{\circ}{x}$ for all $x$ in $X$ is obviously increasing.

For any subset $A$ of $X$, let $A^{*}=\left\{\xi \in w_{0} X: A \in \mathcal{F}\right\}$. If $\mathcal{F}$ is a filter on $X$, let $\xi^{*}$ be the filter on $w_{0} X$ generated by $\left\{F^{*}: F \in \mp\right\}$. The fact that the latter collection is a filter base and other important properties of this set operator follow from the next proposition.

Proposition 1.4 Let $X$ be a $T_{1}$-ordered space.

(a) For all subsets $A, B$ of $X,(A \cap B)^{*}=A^{*} \cap B^{*}$

(b) If $A, B \in C_{X}$, then $((X-A) \cup(X-B))^{*}=(X-A)^{*} \cup(X-B)^{*}$

(c) If $A \in C_{X}$, then $(X-A)^{*}=X^{*}-A^{*}$.

Proof. Statement (a) is clear, and (b) follows from Proposition 1.2; (c) is an easy consequence of (b). I

The topology for $w_{0} X$ is defined by choosing for a subbase of closed sets the collection $\left\{\boldsymbol{A}^{*}: A \in\right.$ $\left.C_{X}\right\}$. If $U \in U_{X}$, then $U$ is a finite intersection of complements of c-sets and $U^{*}$ is open in $w_{0} X$; indeed $\left\{U^{*}: U \in U_{X}\right\}$ is a base for the open sets in $w_{0} X$. In particular, sets of the form $V^{*}$ where $V$ is open and monotone in $X$ form an open subbase for $w_{0} X$. It should be noted that if $V$ is a non-fundamental open set in $X$, it is not generally true that $V^{*}$ is open in $w_{0} X$. The following facts about the topology of $w_{0} X$ will be stated for future reference.

Proposition 1.5 Let $X$ be a $T_{1}$-ordered space.

(a) If $B$ is a monotone closed (respectively, open) set in $X$, then $B^{*}$ is monotone in the same sense and closed (respectively, open) in $w_{0} X$.

(b) If $\xi \in w_{0} X$, then the neighborhood filter $V^{*}(\xi)$ at $\xi$ in $w_{0} X$ has for its filter base $\left\{U^{*}: U \in\right.$ $\left.\exists \cap u_{X}\right\}$.

The next two theorems summarize the main results already known about the Wallman ordered compactification. Proofs for these propositions form the main results of [1] and [3] and the reader is referred to 
these sources for further details. Here we should mention again that the proofs in [1] are formulated in the language of "bifilters", but the translation into "c-filter" terminology presents no difficulties.

Theorem 1.6 For any $T_{1}$-ordered space $X,\left(w_{0} X, \varphi\right)$ is an ordered compactification of $X$, and $w_{0} X$ is a $T_{1}$ topological space. Also, $w_{0} X$ is $T_{2}$-ordered iff $X$ is a $T_{4}$-ordered c-space.

Theorem 1.7 Let $X$ be a $T_{1}$-ordered space, $Y$ a $T_{2}$-ordered compact space, and $f: X \rightarrow Y$ a continuous increasing function. Then there is a unique continuous increasing function $f: w_{0} X \rightarrow Y$ such that $f \circ \varphi=f$.

Let us recall that for a space which admits a $T_{2}$-ordered compactification (see $[5]$ for a characterization of such spaces) there is always a largest $T_{2}$-ordered compactification called the Stone-Čech ordered (or Nachbin) compactification denoted by $\beta_{0} X$ (see [2], [5]). The two preceding theorems yield the following important corollary.

Corollary 1.8 For a space $X$ which admits a $T_{2}$-ordered compactification, $w_{0} X$ and $\beta_{0} X$ are equivalent iff $X$ is a $T_{1}$-ordered c-space.

\section{Separation Properties of $w_{0} X$.}

Given a $T_{1}$-ordered space $X$, we already know that $w_{0} X$ is $T_{1}$, and that $w_{0} X$ is $T_{2}$-ordered iff $X$ is a $T_{4}$-ordered c-space. We shall now examine conditions on $X$ subject to which $w_{0} X$ is $T_{1}$-ordered or $T_{2}$. As it turns out, $w_{0} X$ can fail to have either of these latter properties, can have either one without the other, or can have both properties and still fail to be $T_{2}$-ordered; examples are given later to illustrate all of these possibilities. We begin by finding conditions on $X$ which are necessary and sufficient for $w_{0} X$ to be $T_{2}$.

Proposition 2.1 Let $\mathcal{F}$ be an ultrafilter and $\mathcal{G}$ a maximal c-filter on $X$. Then $\varphi(f) \rightarrow \mathcal{G}$ insor $X$ iff $\xi^{\wedge} \subseteq G$.

Proof. Let $\varphi(\mathcal{F}) \rightarrow \mathcal{G}$ in $w_{0} X$. Let $F \in \mathcal{F}$ be a c-set. If $F \notin \mathcal{G}$ then either $I(F) \notin \mathcal{G}$ or $D(F) \notin \mathcal{G}$; without loss of generality, assume the former. Then $I(F) \notin \mathcal{G}$ implies, by Proposition 1.2, that $X-I(F) \in \mathcal{G}$, and therefore $\mathcal{G} \in(X-I(F))^{*}$, which is a subbasic open neighborhood of $\mathcal{G}$ in $w_{0} X$. Now $\varphi(\mathcal{F}) \rightarrow \mathcal{G}$ implies $(X-I(F))^{*} \in \varphi(\mathcal{F})$, and consequently $X-I(F) \in \mathcal{F}$. This contradicts the fact that $F \in \mathcal{F}$, and therefore every element of $\xi^{\wedge}$ is in $\mathcal{G}$. 
Conversely, let $\mathcal{F}^{\wedge} \subseteq \mathcal{G}$, and $(X-A)^{*}$ be a subbasic open neighborhood of $\mathcal{G}$, where $A$ is closed and monotone in $X$. Since $\mathcal{F}$ is an ultrafilter, either $A \in \mathcal{F}$ or $X-A \in \mathcal{F}$. If $A \in \mathcal{F}$ then $A \in \mathcal{F}$, which in turn implies $A \in \mathcal{G}$, contrary to the fact that $X-A \in \mathcal{G}$. Thus $X-A \in \mathcal{F}$, and hence $(X-A)^{*} \in \varphi(\mathcal{F})$. Since $(X-A)^{*}$ is an arbitrary subbasic open neighborhood of $\mathcal{G}, \varphi(\mathcal{f}) \rightarrow \mathcal{G}$. 1

Theorem 2.2 Let $X$ be a $T_{1}$-ordered space. Then $w_{0} X$ is $T_{2}$ iff, for each ultrafilter $₹$ on $X$, there is a unique maximal c-filter $\mathcal{G}$ on $X$ such that $\xi^{\wedge} \subseteq \mathcal{G}$.

Proof. If $w_{0} X$ is $T_{2}$ and $\xi$ an ultrafilter on $X$, then $\varphi(\xi)$ is an ultrafilter on $w_{0} X$ which must converge to some maximal c-filter $\mathcal{G}$, since $w_{0} X$ is compact. By Proposition $2.1, \mathcal{F}^{\wedge} \subseteq \mathcal{G}$. If there is a different maximal c-filter $K$ with $\xi^{\wedge} \subseteq X$, then $\varphi(\xi)$ would also converge to $K$, contrary to the assumption that $w_{0} X$ is $T_{2}$. Thus $\mathcal{G}$ is unique.

Conversely, assume the uniqueness condition. If $w_{0} X$ is not $T_{2}$, there is a filter $A$ on $w_{0} X$ converging to distinct elements $\mathcal{G}, X$ in $w_{0} X$. Let $\exists$ be an ultrafilter on $X$ finer than the filter generated by $\{A \subseteq X$ : $\left.A^{*} \in A\right\}$. One easily verifies that $\varphi(\mathcal{F})$ must converge to both $\mathcal{G}$ and $\sharp$, which, by Proposition 2.1, violates our assumed uniqueness condition. I

A space $X$ is defined to be c-normally ordered if, for each pair of disjoint c-sets $A, B$, there are disjoint fundamental open sets $U, V$ such that $A \subseteq U$ and $B \subseteq V$. As we shall see in later examples, there are spaces which are c-normally ordered but not normally ordered, and vice versa. Of course, both of these versions of "ordered normality" reduce to ordinary normality when the partial order for $X$ is equality.

Theorem 2.9 The following conditions on a $T_{1}$-ordered space $\mathrm{X}$ are equivalent.

(1) $X$ is c-normally ordered.

(2) Two disjoint fundamental closed sets in $X$ can be separated by disjoint fundamental open neighborhoods.

(3) If $A$ is a c-set in $X$, then every fundamental open set containing $A$ contains a fundamental closed set which in turn contains a fundamental open neighborhood of $A$.

(4) For each ultrafilter $\mathcal{F}$ on $X$, there is a unique maximal c-filter $\mathcal{G}$ finer than $\mathcal{F}^{\wedge}$.

(5) $w_{0} X$ is $T_{2}$.

Proof. The equivalence of (1), (2), and (3) is a routine exercise, and the equivalence of (4) and (5) was established in the previous theorem. 
(1) $\Rightarrow(5)$. If $\mathcal{F}$ and $\mathcal{G}$ are distinct maximal c-filters on $X$, then there are disjoint c-sets $F$ in $\mathcal{F}$ and $G \in G$. Let $U$ and $V$ be disjoint fundamental open neighborhoods of $F$ and $G$ respectively; then by Proposition 1.4, $U^{*}$ and $V^{*}$ are disjoint open neighborhoods of $\mathcal{F}$ and $\mathcal{G}$, respectively, in $w_{0} X$.

(5) $\Rightarrow(1)$. Let $A$ and $B$ be disjoint c-sets in $X$. Then $A^{*}$ and $B^{*}$ are disjoint closed sets in $w_{0} X$, and since $w_{0} X$ is compact and $T_{2}$, there are disjoint open sets $M$ and $N$ in $w_{0} X$ such that $A^{*} \subseteq M$ and $B^{*} \subseteq N$. Since $\left\{U^{*}: U \in U_{X}\right\}$ forms an open base for $w_{0} X$, there are subcollections $\left\{U_{i}^{*}: i \in I\right\}$ and $\left\{V_{j}^{*}: j \in J\right\}$ such that $M=U\left\{U_{i}^{*}: i \in I\right\}$ and $N=U\left\{V_{j}^{*}: j \in J\right\}$. Using the fact that $A^{*}$ and $B^{*}$ are compact subsets in $w_{0} X$, we can find finite subcovers $U_{i_{1}}^{*}, \cdots, U_{i_{n}}^{*}$ of $A^{*}$ and $V_{j_{1}}^{*}, \cdots, V_{j_{m}}^{*}$ of $B^{*}$. Letting $U=U_{i_{1}} \cup \cdots \cup U_{i_{n}}$ and $V=U_{j_{1}} \cup \cdots \cup U_{j_{m}}$, we obtain disjoint fundamental open neighborhoods of $A$ and $B$ in X. I

Although neither of the properties "normally ordered" and "c-normally ordered" implies the other in general, the next theorem establishes the equivalence of these properties in $T_{1}$-ordered c-spaces. We first need the following lemma.

Lemma 2.4 Let $X$ be a c-normally ordered c-space. If $A$ is a c-set in $X$ and $U$ is an open, increasing neighborhood of $A$, then there is a closed, increasing neighborhood $G$ of $A$ such that $A \subseteq G \subseteq U$.

Proof. Let $B=X-U$; by Proposition 1.3, $I(A) \cap B=\phi$, and so $I(A)$ and $B$ can be separated by disjoint, fundamental open sets $W$ and $V$, respectively. By Proposition $1.1, X-V$ is a finite union of c-sets $C_{1}, \cdots, C_{n}$. By Proposition 1.3, $I\left(C_{i}\right) \cap B=\phi$ for all indices $i$; let $G=\cup\left\{I\left(C_{i}\right): i=1, \cdots, n\right\}$. Thus $G$ is closed and increasing, and $A \subseteq W \subseteq G \subseteq U$.।

Theorem 2.5 For a $T_{1}$-ordered c-space $X$, the following statements are equivalent.

(a) $X$ is normally ordered.

(b) $X$ is c-normally ordered.

(c) $w_{0} X$ is $\mathrm{T}_{2}$-ordered.

Proof. (a) $\Leftrightarrow$ (c) is established in Theorem 1.6. (c) $\Rightarrow$ (b) follows by Theorem 2.3. (b) $\Rightarrow$ (c): It is sufficient to show that if $\xi, G \in w_{0} X$ and $\xi\{G$, then there are disjoint neighborhoods of $\exists$ and $G$ in $w_{0} X$, where the former is an increasing set and the latter decreasing. If $₹\{g$, then either $I(f) \nsubseteq \mathcal{G}$ or $D(\mathcal{G}) \nsubseteq \mathcal{F}$; without loss of generality, assume the latter. Since $\mathcal{I}$ is a maximal c-filter, there are c-sets $G \in \mathcal{G}$ and $F \in \mathcal{F}$ such that $D(G) \cap F=\phi$. By Lemma 2.4, there is a closed, increasing neighborhood $N$ of $F$ such that $N \cap D(G)=\phi$ and a fundamental open set $W$ such that $F \subseteq W \subseteq N$. Now $(X-N)^{*}$, which is 
a decreasing open set in $w_{0} X$ by Proposition 1.5 , and the increasing hull of $W^{*}$ in $w_{0} X$ provide the desired neighborhoods which separate $\mathcal{F}$ and $\mathcal{G}$ in $w_{0} X .1$

If $w_{0} X$ is $T_{2}$-ordered, then $X$ is necessarily a $T_{1}$-ordered c-space; thus the following corollary is immediate.

Corollary 2.6 For a $T_{1}$ - ordered space $X, w_{0} X$ is $T_{2}$-ordered iff $X$ is a c-normally ordered c-space.

Theorem 2.7 If $X$ is a $T_{1}$-ordered c-space, then $w_{0} X$ is $T_{1}$-ordered.

Proof. Let $J \in w_{0} X$, and let $i_{w}(\mathcal{F})=\left\{\mathcal{G} \in w_{0} X: \mathcal{J} \mathcal{g}\right\}$ be the increasing hull of $\mathcal{F}$ in $w_{0} X$. Let "cl $l_{w}$ denote the closure operator in $w_{0} X$. We shall show that $c l_{w}\left(i_{w}(f)\right) \subseteq i_{w}(f) ;$ a similar argument shows that the decreasing hull of $J$ is closed, and hence that $w_{0} X$ is $T_{1}$-ordered.

If $\mathcal{G} \in c l_{w}\left(i_{w}(\mathcal{f})\right)$, then for each $A \in C_{X}$ such that $\mathcal{G} \in(X-A)^{*}$, there is $\forall \in i_{w}(\mathcal{f})$ such that $\forall \in(X-A)^{*}$. With the help of Proposition 1.2, the last sentence may be restated as follows: if $G \in C l_{w}\left(i_{w}(\mathcal{F})\right)$ and $A \in C_{X}$, then $A \notin G$ implies there is $\forall \in w_{0} X$ such that $₹ \lesssim \forall$ and $A \notin \psi$.

Now assume that $\mathcal{G} \in c_{w}\left(i_{w}(\mathcal{F})\right)$; if $\mathcal{G} \notin i_{w}(\mathcal{F})$, then either $I(\mathcal{F}) \nsubseteq \mathcal{G}$ or $D(\mathcal{G}) \nsubseteq \mathcal{F}$. Suppose $I(\mathcal{F}) \notin \mathcal{G}$; then there is $F \in \mathcal{F}$ such that $I(F) \notin \mathcal{G}$. But $\mathcal{G} \in \mathrm{cl}_{w}\left(i_{w}(\mathcal{F})\right)$ implies there is $k \in i_{w}(\mathcal{F})$ such that $I(F) \notin \mathcal{X}$, a contradiction. On the other hand, suppose $D(\mathcal{G}) \notin \mathcal{F}$. Since $\mathcal{F}$ is a maximal c. filter, there are c-sets $F \in \mathcal{J}$ and $G \in \mathcal{G}$ such that $D(G) \cap F=\phi$, and by Proposition 1.3, $D(G) \cap I(F)=\phi$. Thus $I(F) \notin \mathcal{G}$, and a repetition of the preceding argument again yields a contradiction. We may therefore conclude that $\mathcal{G} \in i_{w}(\mathcal{F})$, and hence that $i_{\varpi}(\mathcal{F})$ is closed. $I$

The converse of Theorem 2.7 does not hold in general, however the next theorem establishes a partial converse. We shall say that a net $\left(x_{\lambda}\right)_{\lambda \in \Lambda}$ in a space $X$ is upward directed if, for each pair of indices $\lambda, \mu \in \Lambda$, there is $\sigma \in \Lambda$ such that $\lambda \leq \sigma, \mu \leq \sigma, x_{\lambda} \leq x_{\sigma}$, and $x_{\mu} \leq x_{\sigma}$. Downsward directed nets are defined dually.

Theorem 2.8 Let $X$ be a $T_{2}$-ordered space with the property that, whenever $A$ is decreasing (respectively increasing) and $x \in c l_{X} A$, there is an upward directed (respectively, downward directed) net on $A$ which converges to $x$. Then $w_{0} X$ is $T_{1}$-ordered iff $X$ is a c-space.

Proof. Suppose $X$ is not a c-space. Then for some c-set $A$ in $X$, either $i(A)$ is not closed or else $d(A)$ is not closed. There is no loss of generality in assuming the latter. Thus there is some $y \in \operatorname{cl} X d(A)$ such that $y \notin d(A)$; by assumption there is an upward directed net $\left(x_{\lambda}\right)_{\lambda \in A}$ on $d(A)$ converging to $y$. Then $\left\{i\left(x_{\lambda}\right) \cap A: \lambda \in \Lambda\right\}$ is a base for a c-filter $\mathcal{G}$ on $X$; let $\mathcal{F}$ be a maximal c-filter finer than $\mathcal{G}$. 
Let $K$ be the filter generated by the net $\left(x_{\lambda}\right)_{\lambda \in \Lambda}$. Then $K \rightarrow y$, and if $z \rightarrow x$ for some $x \in X$, then it must follow that $y \leq x$, since $K \times \mp$ has a trace on the order, and the order is closed. But $y \leq x$ is a contradiction since $A \in \mathcal{F}$ and $A$ a $c$-set implies $x \in A$, and therefore $y \in d(A)$. Thus $\mathcal{F}$ must be a non-convergent maximal $c$-filter, and therefore an element of $w_{0} X$.

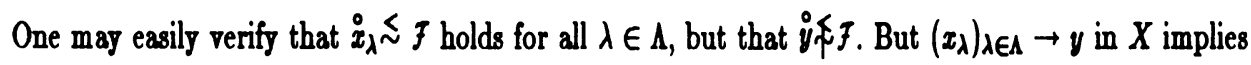
$\left(\stackrel{0}{x}_{\lambda}\right)_{\lambda \in \Lambda} \rightarrow \mathfrak{y}$ in $w_{0} X$, and hence $\dot{y} \in c l_{w}\left(d_{w}(f)\right)$, but $\dot{y} \notin d_{w}(f)$. Thus $w_{0} X$ is not $T_{1}$-ordered. I

Example 2.9 Let $X=A \cup B \cup\{a\} \cup\{b\}$, where $A=\left\{x_{i}: i=1,2,3, \cdots\right\}$ and $B=\left\{y_{i}: i=1,2,3, \cdots\right\}$. Define the topology for $X$ by specifying that $\{x\}$ is open for $x \in A \cup B$; the neighborhood filter at a (respectively, $b$ ) is generated by sets of the form $A_{n}=\left\{x_{i}: i \geq n\right\}$ (respectively, $B_{n}=\left\{y_{i}: i \geq n\right\}$ ), where $n=1,2,3, \cdots$. The order for $X$ is the smallest partial order such that $x_{i} \leq y_{i}$ for each positive integer $i$.

It is evident from this construction that $X$ is a compact, $T_{2}, T_{1}$-ordered space; thus we may identify $X$ with $w_{0} X$. Note that every closed set in $X$ is a c-set, and every open set is a fundamental open set; it follows that $X$ is c-normally ordered. However $X$ is not a c-space, since for the c-set $C=B \cup\{b\}, i(C)=A \cup C$ is not closed. Thus $X$ is neither $T_{2}$-ordered nor normally ordered.

The main points illustrated by this example are that the converse of Theorem 2.7 does not hold in general, and that the conditions $T_{1}$-ordered, $T_{2}$, and c-normally ordered on $X$ are not sufficient to guarantee that $w_{0} X$ is $T_{2}$-ordered. This example also shows that a c-normally ordered space need not be normally ordered, even when the axioms $T_{1}$-ordered and $T_{2}$ are present.

\section{Examples in Euclidean Space.}

Additional insight into the behavior of the Wallman ordered compactification can be gained by study ing some simple examples in $R^{n}$ (by which we mean Euclidean n-space with the usual product topology and product order), especially in the case $n=2$. We shall show that $n=2$ is the largest value of $n$ for which $w_{0} R^{n}$ is $T_{2}$-ordered, and hence the largest value of $n$ for which $w_{0} R^{n}=\beta_{0} R^{n}$. We shall use the known properties $w_{0} R^{2}$ to describe $\beta_{0} R^{2}$. We also examine two simple subspaces of $R^{2}$ for which the Wallman ordered compactification is not $T_{2}$-ordered.

Theorem 9.1 Let $A$ be a closed, convex subset of $R^{2}$. Then $i(A)=I(A)$ and $d(A)=D(A)$, and hence $R^{2}$ is a c-space. 
Proof. We shall prove that $i(A)$ is closed; a similar argument shows that $d(A)$ is closed.

If $i(A)$ is not closed, then there is a sequence $\left(x_{n}, y_{n}\right)$ in $i(A)$ such that $\left(x_{n}, y_{n}\right) \rightarrow\left(x_{0}, y_{0}\right)$, where $\left(x_{0}, y_{0}\right) \notin i(A)$. Let $\left(a_{n}, b_{n}\right)$ be a sequence in $A$ such that $\left(a_{n}, b_{n}\right) \leq\left(x_{n}, y_{n}\right)$ for all $n \in Z^{+}$. The convergence of the sequence $\left(x_{n}, y_{n}\right)$ implies that the sequences $\left(a_{n}\right)$ and $\left(b_{n}\right)$ are both bounded above. Either of these sequences may fail to be bounded below, and this leads us to consider four cases.

Case 1. $\left(a_{n}\right)$ and $\left(b_{n}\right)$ are both bounded below. Then there is a convergent subsequence $\left(a_{n_{k}}, b_{n_{k}}\right) \rightarrow$ $(a, b)$. Since $A$ is closed, $(a, b) \in A$, and since $R^{2}$ is $T_{2}$-ordered, $(a, b) \leq\left(x_{0}, y_{0}\right)$, contrary to $\left(x_{0}, y_{0}\right) \notin i(A)$.

Case 2. $\left(a_{n}\right)$ and $\left(b_{n}\right)$ are both unbounded below. Then for some $n \in Z^{+},\left(a_{n}, b_{n}\right) \leq\left(x_{0}, y_{0}\right)$, which again contradicts $\left(x_{0}, y_{0}\right) \notin i(A)$.

Case 9. $\left(a_{n}\right)$ is bounded below, but $\left(b_{n}\right)$ is not. In this case, there is no loss of generality in assuming that $a_{n} \rightarrow a$ and that $\left(b_{n}\right)$ is an unbounded, decreasing sequence. Then there is $n_{0} \in Z^{+}$such that $b_{n} \leq y_{0}$, for all $n \geq n_{0}$. Also $a \leq x_{0}$ since $R^{1}$ is $T_{2}$-ordered, and for $n \geq n_{0}$ we must have $a \leq a_{n}$, for otherwise $\left(a_{n}, b_{n}\right) \leq\left(x_{0}, y_{0}\right)$ would again yield a contradiction. Thus from the sequence $\left(a_{n}\right)_{n \geq n_{0}}$ it is possible to obtain a decreasing subsequence $\left(a_{n_{k}}\right) \rightarrow a$, and the corresponding subsequence $\left(b_{n_{k}}\right)$ is, of course, decreasing and unbounded. Now for any $j \in Z^{+},\left(a_{n_{j}}, b_{n_{j}}\right) \leq\left(a_{n_{j}}, b_{n_{1}}\right) \leq\left(a_{n_{1}}, b_{n_{1}}\right)$, and the convexity of $A$ implies that $\left(a_{n_{j}}, b_{n_{1}}\right) \in A$ for all $j \in Z^{+}$. Thus, $\left(a_{n_{j}}, b_{n_{1}}\right) \rightarrow\left(a, b_{n_{1}}\right)$ and $\left(a, b_{n_{1}}\right) \in A$ since $A$ is closed. But $a \leq x_{0}$ and $b_{n_{1}} \leq y_{0}$ implies $\left(x_{0}, y_{0}\right) \in i(A)$, a contradiction.

Case f. $\left(b_{n}\right)$ is bounded below, but $\left(a_{n}\right)$ is not. An argument similar to that of case 3 yields a contradiction. I

Proposition 9.2 $R^{n}$ is not a c-space for $n \geq 3$.

Proof. Let $A=\left\{\left(m,-\frac{1}{m}, \frac{1}{m}, 0, \cdots, 0\right) \in R^{n}: m \in Z^{+}\right\}$. The elements of $A$ are isolated in both the topological and order sense, and so $A$ is a closed, convex subset of $R^{n}$. One can verify that $I(A)=i(A)$ and $D(A)=d(A) \cup B$, where $B=\left\{(x, 0, z, 0, \cdots, 0) \in R^{n}: z \leq 0\right\}$. Since $i(A) \cap B=\phi$, it follows that $A=I(A) \cap D(A)=A^{\wedge}$, and so $A$ is a c-set. But $d(A) \neq D(A)$, and so $R^{n}$ is not a c-space. I

Theorem $9.9 R^{n}$ is $T_{4}$-ordered for all $n \in Z^{+}$.

Proof. If $x=\left(x_{1}, \cdots, x_{n}\right) \in R^{n}$ and $x \leq y$, then $N(x, \varepsilon) \subseteq d(N(y, \varepsilon))$ and $N(y, \varepsilon) \subseteq i(N(x, \varepsilon)) ;$ from this it easily follows that the increasing and decreasing hulls of open sets in $R^{n}$ are open. If $A$ is a closed increasing set and $B$ a closed, decreasing set in $R^{n}$ such that $A \cap B=\phi$, then for each $b \in B$ we may choose 
$r_{b}$ such that $A \cap N\left(b, r_{b}\right)=\phi$, and consequently $A \cap d\left(N\left(b, r_{b}\right)\right)=\phi$. Likewise, for each $a \in A$, there is $r_{a}$ such that $B \cap i\left(N\left(a, r_{a}\right)\right)=\phi$. Let $U=\cap\left\{i\left(N\left(a, r_{a} / 2\right)\right): a \in A\right\}$ and $V=U\left\{d\left(N\left(b, r_{b} / 2\right)\right): b \in B\right\}$. Then $U$ and $V$ are disjoint open sets, the former increasing and the latter decreasing, which separate $A$ and $B . I$

Theorem 9.f The following statements are equivalent.

(a) $R^{n}$ is a c-space.

(b) $w_{0} R^{n}$ is $T_{1}$-ordered.

(c) $w_{0} R^{n}$ is $T_{2}$-ordered.

(d) $w_{0} R^{n}=\beta_{0} R^{n}$.

(e) $n \leq 2$.

Proof. It is obvious that in $R^{1}$, the increasing or decreasing hull of any closed set is closed, and so $R^{1}$ is a c-space. Thus (a) $\Leftrightarrow$ (c) follows by Theorem 3.1 and Proposition 3.2.

(a) $\Leftrightarrow$ (b) follows from Theorems 2.7 and 2.8. By Theorems 1.6, 3.1, and 3.3, $w_{0} R^{n}$ is $T_{2}$-ordered for $n \leq 2$, and by Theorem 1.6 and Proposition 3.2, $w_{0} R^{n}$ is not $T_{2}$-ordered for $n \geq 3$; thus (c) $\Leftrightarrow$ (e). Finally, (c) $\Leftrightarrow$ (d) follows by Theorem 1.6 and Corollary 1.8.

The Wallman ordered compactification of $R^{1}$ is the familiar two point compactification which is commonly called the "extended real line." In the case of $R^{2}$, this compactification, which is not so familiar, is described in the next example.

Example $9.5 R^{2}$ is simultaneously homeomorphic and order isomorphic to the open square $S=$ $\left\{\left(x_{1}, x_{2}\right) \in R^{2}:-1<x_{1}<1,-1<x_{2}<1\right\}$. The closed square $S=\left\{\left(x_{1}, x_{2}\right) \in R^{2}:-1 \leq x_{1} \leq\right.$ $\left.1,-1 \leq x_{2} \leq 1\right\}$ can thus be regarded as a $T_{2}$-ordered compactification of $R^{2}$. The most convenient way to describe $w_{0} R^{2}$ (or, equivalently, $\beta_{0} R^{2}$ ) is to is to consider each boundary (i.e., compactification) point $p$ of $\bar{S}$ to be replaced by the set $M_{p}$ of all non convergent maximal c filters $\forall$ on $S$ which converge to $p$ in $\bar{S}$. If $p=(1,1)$, then $M_{p}$ consists of a single maximal $c$-filter which is the greatest element of $w_{0} R^{2}$. Similarly, the least element of $w_{0} R^{2}$ is the unique maximal c-filter in $M_{(-1,-1)}$. If $p$ is any boundary point of $S$ other than $(1,1)$ or $(-1,-1)$, then $M_{p}$ contains $2^{\gamma}$ distinct elements, where $\gamma$ is the cardinality of the real line, including both a greatest and a least element. For instance, if $p=(1,0)$ the least element in $M_{p}$ is a maximal c-filter in $S$ which contains the positive $x$ axis and converges in $S$ to $(1,0)$; the greatest element is a maximal c-filter finer than the filter supremum of $\left\{I(\mathcal{F}): \mathcal{F} \in \mathcal{M}_{p}\right\}$ which converges to $(1,0)$ in $S$. 
If $p, q$ are two boundary points in $\bar{S}$ and $p \leq q$ in $\bar{S}$, then $G \lesssim \forall$ for all $G \in M_{p}$ and for all $\forall \in M_{q}$; furthermore if $\mathcal{G} \lesssim \forall$ for some $\mathcal{G} \in M_{p}$ and for some $\forall \in M_{q}$, then $p \leq q$ in $\bar{S}$. Similarly if $x \in S$ and $p$ is a boundary point of $\bar{S}$, then $x \leq p$ in $\bar{S}$ iff $i \lesssim \forall$ for some $\forall \in M_{p}$ (in which case $i \lesssim \forall$ for all $\forall \in M_{p}$ ). I

The next two examples show that even for the simplest subspaces $X$ of $R^{2}$, various pathologies can arise in $w_{0} X$.

Example S.6 Let $X_{1}$ be the closed square $S$ (defined in Example 3.5) with the origin $(0,0)$ deleted, and with the topology and order inherited from $R^{2}$. Let $\mathcal{G}$ (respectively, $\not$ ) be the maximal c-filter on $X_{1}$ which contains the negative portion of the $x$-axis (respectively, $y$-axis) and converges to $(0,0)$ in $S$. If $A=\{(x, 0): x<0\}$ and $B=\{(0, y): y<0\}$, then $A$ and $B$ are c-sets in $X_{1}$. Since $B \subseteq D(A)$ but $B \cap d(A)=\phi, d(A) \neq D(A)$ and thus $X_{1}$ is not a c-space. Furthermore, it follows from Theorem 2.8 that $w_{0} X_{1}$ is not $T_{1}$-ordered. Also, $A$ and $B$ cannot be separated by fundamental open sets, and consequently $w_{0} X_{1}$ is not $T_{2}$. However, the argument used to prove Theorem 3.3 can be applied to show that $X_{1}$ is $T_{4}$ ordered. We thus have an example which, in contrast to Example 2.9 , is normally ordered but not c-normally ordered, and for which the Wallman ordered compactification is neither $T_{1}$-ordered nor $T_{2}$.

It is easy to describe $w_{0} X_{1}$. The "hole" at $(0,0)$ in $X_{1}$ is filled in $w_{0} X_{1}$ by a set $M_{(0,0)}$ of maximal c.filters on $X_{1}$ which converge to $(0,0)$ in $\bar{S}$. The filters $\mathcal{G}$ and $\forall$ described above are minimal elements in $M_{(0,0)}$; there are also two maximal elements in $M_{(0,0)}$ which are maximal c-filters converging to $(0,0)$ in $\bar{S}$ along the positive $x$ and $y$ axes. The set of compactification points contains no greatest or least element and has cardinality 27.1

Example 9.7 Let $X_{2}$ be the closed square $S$ with the $y$ axis deleted except for the origin; the topology and order are those inherited from $R^{2}$. One may show that this space is both $T_{4}$-ordered and c-normally ordered. However, $X_{2}$ is not a c-space, for if $A=\left\{\left(x, \frac{1}{2}\right): x<0\right\}$, then $A$ is a c-set and $(0,0) \in D(A)$. Thus, $w_{0} X_{2}$ is $T_{2}$ by Theorem 2.3, but $w_{0} X_{2}$ is not $T_{1}$-ordered by Theorem 2.8. Without going into detail, we can partially describe $w_{0} X_{2}$ by remarking that every "hole" in $X_{2}$ corresponding to a missing point on the $y$-axis is filled in $v_{0} X_{2}$ by adding $2^{\gamma}$ compactification points including, in each case, a pair of minimal elements and a pair of maximal elements. I

For the sake of completeness, we should give an example of a space $X$ for which $w_{0} X$ is $T_{1}$-ordered but not $T_{2}$. This turns out to be quite easy. Let $X$ be any $T_{2}$, completely regular toplogical space which is 
not normal, and let the order for $X$ be equality. Then it is well known that $w_{0} X$ is $T_{1}$ (and hence $T_{1}$-ordered) but not $T_{2}$.

Our final example does not pertain directly to the Wallman ordered compactification, but it does provide further insight into the nature of c-sets, which ase crucial ingredients in the construction of this compactification. It shows that closed, convex subsets of $R^{3}$ need not be c-sets, and that the relation defined by " $A$ is a c-set in $B$ " is not transitive. We are grateful to Dr. Bettina Zoeller for providing this example as well as the related example used in the proof of Proposition 3.2.

Example 9.8 Let $K=\left\{\left(m,-\frac{1}{m}, \frac{1}{m}\right): m \in Z^{+}\right\} \cup\left\{\left(-m, \frac{1}{m},-\frac{1}{m}\right): m \in Z^{+}\right\}$be a subset of $R^{3}$; note that $K$ is closed and convex. Let $L=I(K) \cap D(K)$; then $L$ is the union of $K$ with the $x$-axis, and consequently $K$ is not a c-set. Furthermore, observe that $K$ is a c-set in $L$ (considered as a subspace of $R^{3}$ ) and $L$ is a c-set in $R^{3}$, but $K$ is not a c-set in $R^{3}$.

Such an example cannot be found in $R^{n}$ for $n \leq 2$, since in these spaces every closed, convex set is a c-set.

\section{References}

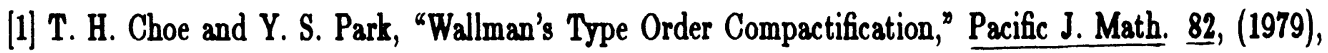
339347.

[2] P. Fletcher and W. Lindgren, Quasi-Uniform Spaces, Lect. Notes in Pure and Appl. Math., Vol. 77 (1982).

[3] D. C. Kent, "On the Wallman Order Compactification," Pacific J. Math. 118 (1985), 159163.

[4] S. D. McCartan, "Separation Axioms for Topological Ordered Spaces," Proc. Camb. Phil. Soc. 64 (1968), 965973.

[5] L. Nachbin, Topology and Order, Van Nostrand Mathematical Series 4, Princeton, N.J. 1965.

[6] H. A. Priestly, "Ordered Topological Spaces and Representation of Distributive Lattices," Proc. London Math. Soc. 24 (1972), 507530. 


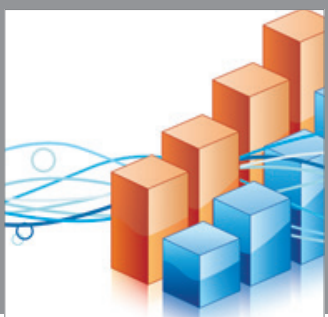

Advances in

Operations Research

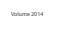

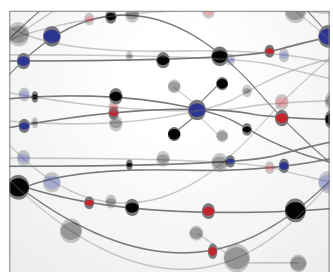

\section{The Scientific} World Journal
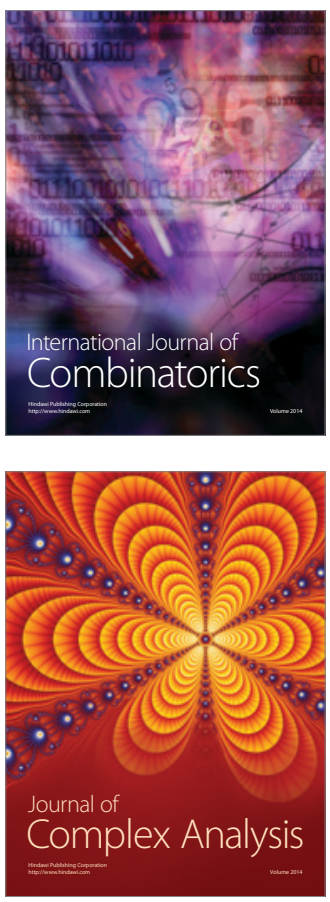

International Journal of

Mathematics and

Mathematical

Sciences
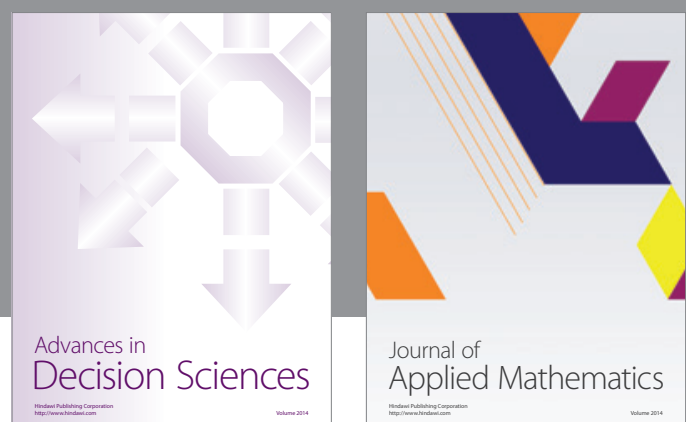

Journal of

Applied Mathematics
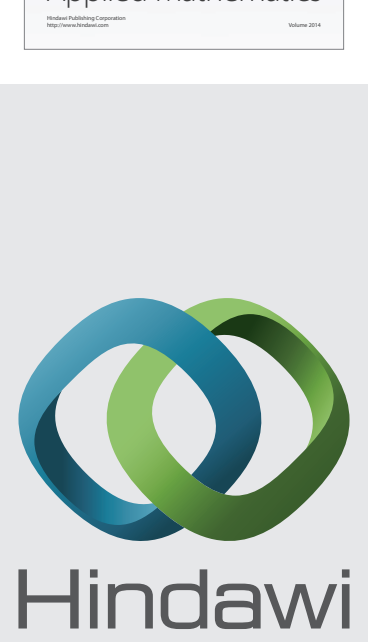

Submit your manuscripts at http://www.hindawi.com
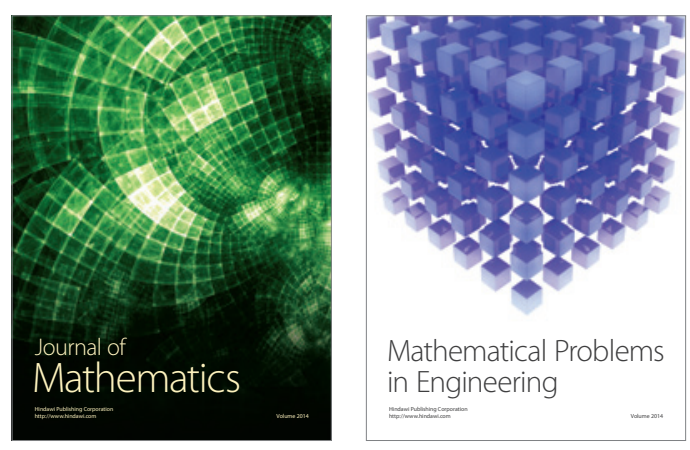

Mathematical Problems in Engineering
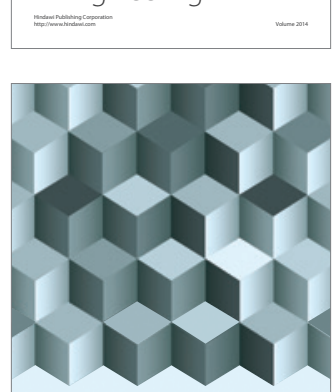

Journal of

Function Spaces
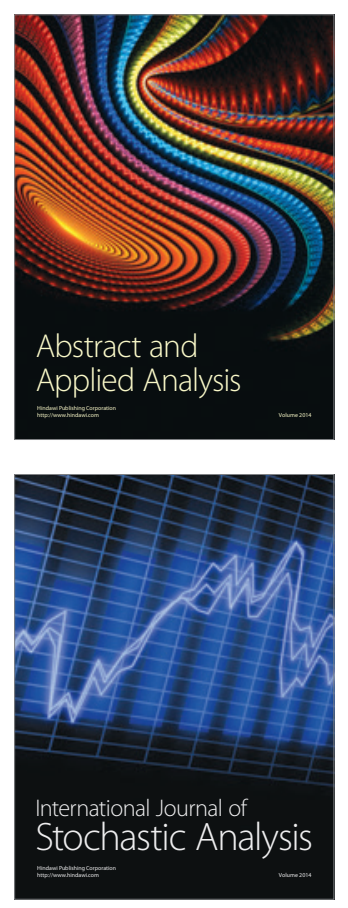

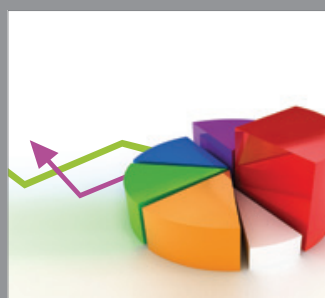

ournal of

Probability and Statistics

Promensencen
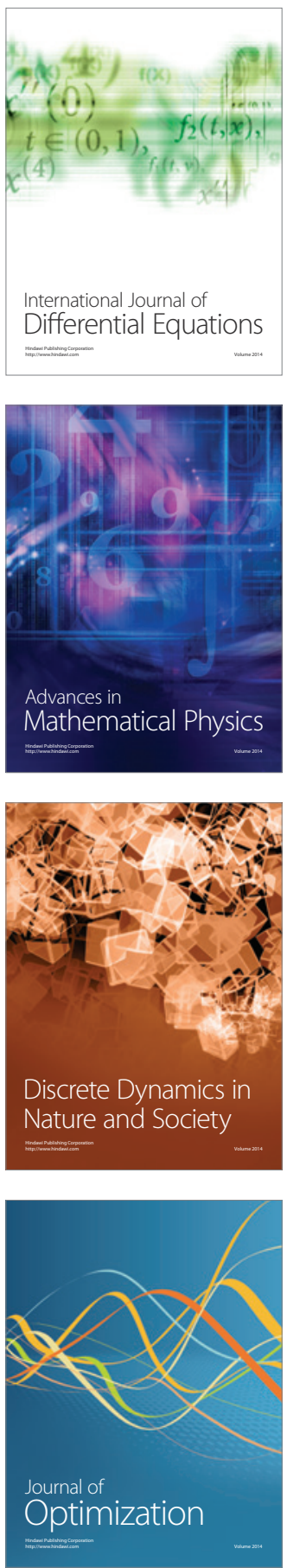